\title{
Welches Essen verhindert Diabetes am besten?
}

Fragestellung: Welche Nahrungsmittel sind am besten geeignet, um eine Reduktion des Typ-2-Diabetes-Risikos zu unterstützen - Obst, Gemüse, Fisch und Meeresfrüchte oder Milch?

Hintergrund: Es gibt eine immer stärker wachsende kontroverse Diskussion, welche Art der Ernährung am besten einem Typ2-Diabetes vorbeugt. Der umgekehrte Effekt ist gut bekannt, dass viele industriell hergestellte Nahrungsmittel über eine Veränderung des Mikrobioms im Darm das Risiko von chronischen Erkrankungen ganz drastisch beeinflussen können. Geht das

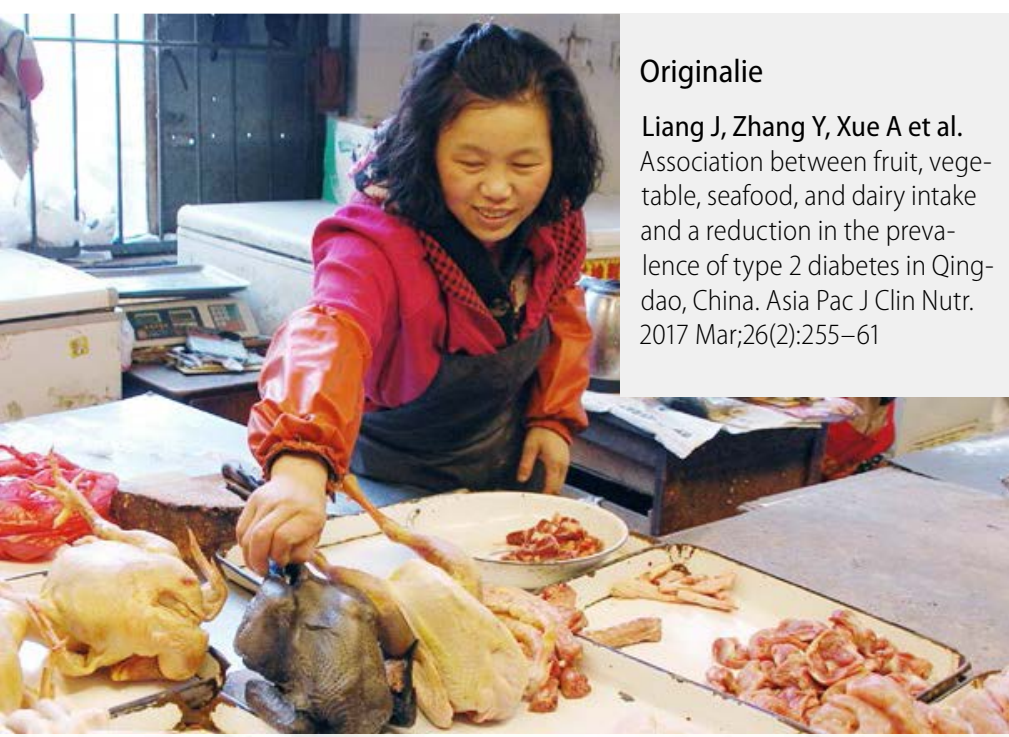

Essen Frauen in China statt Fleisch mehr Joghurt, senkt das ihr Diabetesrisiko drastisch. Bei Männern hat sea food diesen Effekt. aber auch vice versa - also können bestimmte Nahrungsmittel stärker diabetesvorbeugend sein als andere? In einer Zeit, in der wir immer mehr industriell hergestellte sowie preis- und damit oft ungünstige Lebensmittel konsumieren, wird diese Frage immer wichtiger. Manche Forscher behaupten, dass wir mittlerweile in einem „toxic food environment“ leben. Wenn das stimmt, muss es aber auch den umgekehrten Effekt geben.

Patienten und Methoden: Insgesamt wurden 4.343 Personen in China im Alter zwischen 35 und 74 Jahren in eine bevölkerungsbasierte Querschnittstudie einbezogen. Standardisierte Ernährungsfragebögen wurden genutzt, um die Menge und Häufigkeit von Obst, Gemüse, Meeresgetier und Milchverbrauch zu bestimmen. Die Diabetesdiagnose wurde anhand von WHO/IDF-2006-Kriterien bestimmt. Mithilfe logistischer Regressionsanalysen wurde das Diabetesrisiko in Abhängigkeit vom Ernährungsverhalten bestimmt.

Ergebnisse: Das relative Risiko für Diabetes war in der Kohorte in Abhängigkeit vom Obstkonsum 0,86, Gemüse 0,50 und der Kombination aus beiden 0,91 bei Frauen. Der stärkere Verbrauch von Milch- und Joghurtprodukten reduzierte das Diabetesrisiko auf 0,56 bei Frauen. Alle diese Effekte waren bei Männern nicht zu beobachten. Interessanterweise zeigte sich aber bei Männern durch den Konsum von Meeresprodukten eine Reduktion des Diabetesrisikos auf 0,58.

Schlussfolgerungen: Die Autoren schlussfolgern daher, dass Obst, Gemüse und Joghurt/Milchverbrauch bei Frauen Diabetes verhindert. Aber das alles gilt für die chinesische Population. Die Autoren schlagen vor, dass die entsprechenden Ergebnisse in prospektiven Studien bestätigt werden müssen.

\section{- Kommentar von Prof. Dr. med. Peter E. H. Schwarz}

\section{Es ist Zeit für eine prospektive Studie zu gesunder Ernährung in Asien}

Verglichen mit Asien oder den arabischen Staaten ist Deutschland fast diabetesfrei. Die Zunahme der Diabetesprävalenz in diesen Ländern verläuft deutlich schneller und aggressiver als in Europa. Aus diesem Grund ist es logisch zu untersuchen, welche Verhaltensweisen das Diabetesrisiko dort reduzieren können. Die Fragestellung nach Obst, Gemüse, Meeresfrüchten und Milch mag veraltet erscheinen, aber ist in diesen Ländern von hoher Relevanz. Verblüffend sind die Ergebnisse, dass eine gesunde Obst-Milch-Ernährung bei Frauen wirkungsvoll ist, bei Männern aber das Essen von Meeresgetier.

Über die Gründe dafür kann nur spekuliert werden, vermutlich ist es bei Männern das Switchen von einem starken Fleischkonsum zu Fischkonsum, was den entscheidenden Effekt hat. Dieses Ergebnis wäre nicht verwunderlich. Beeindruckend in der Studie ist allerdings die starke Risikoreduktion bei Frauen durch eine gesunde Ernährung. Die Ergebnisse sind dort fast stärker als in europäischen und amerikanischen Studien. Das sollte zu einer prospektiven Studie zum Thema in der asiatischen Bevölkerung anstiften. Für Kaukasier liegen diese vor. Es ist zu vermuten, dass auch in Asien gezeigt werden kann, dass eine gesunde Ernährung Diabetes verhindert.

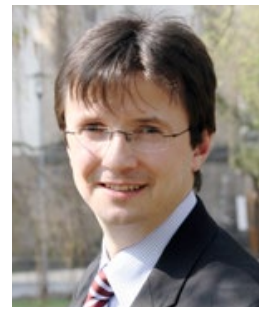

Prof. Dr. med. Peter E. H. Schwarz

Med. Klinik III, TU Dresden, Fetscherstraße 74, 01307 Dresden und Paul Langerhans Institut Dresden, German Center for Diabetes Research peter.schwarz@uniklinikum-dresden.de 